Series A

I. MATHEMATICA

418

\title{
ON THE USE OF STEP-FUNCTIONS IN EXTREMUM PROBLEMS OF THE CLASS WITH BOUNDED BOUNDARY ROTATION
}

BY

H. LONKA and O. TAMMI

H E L S I K I 1968

S U O M A L A I N E T T E DEAKA T E M I A

doi:10.5186/aasfm.1968.418 
Communicated 10 November 1967 by K. I. Virtanen and Lauri Myrberg 


\section{§ 1. On the use of step-functions in $S_{k}$}

We wish to discuss in detail a method of extremalization which is based on the use of step-functions. Let us consider a subclass of univalent functions, the class $S_{k}$, which although rather simple, displays features typical of extremum problems. This class consists of the normalized functions

$$
f(z)=z+a_{\mathbf{2}} z^{2}+\ldots,
$$

regular in the open disc $|z|<1$, and of which the boundary rotation is bounded by the number $k \pi$, where $2 \leq k \leq 4$. According to PaAtero, the functions of $S_{k}$ are univalent [3].

The functions of the class $S_{k}$ can be generated by means of functions $\psi$ of bounded variation in the following sense:

The class $S_{k}$ consists of those functions $f$ which satisfy the PoissonStieltjes equation

$$
1+z \frac{f^{\prime \prime}(z)}{f^{\prime}(z)}=\frac{1}{2} \int_{0}^{2 \pi} \frac{e^{i_{\gamma}}+z}{e^{i_{i}}-z} d \psi(\varphi), \quad|z|<1,
$$

where

$$
\int_{0}^{2 \pi} d \psi(\varphi)=2, \int_{0}^{2 \pi} d \psi(\varphi) \leq k, \quad 2 \leq k \leq 4 .
$$

In what follows, $\Phi_{k}$ denotes the space of all functions $\psi$ of bounded variation, defined on the interval $I=[0,2 \pi]$ and satisfying the conditions (3).

By using the relation (2), the coefficients $a_{p}$ in series (1) of $f(z)$ can be expressed as functionals $a_{p}(\psi)$ of the generating function $\psi$. Thus, for example

$$
\begin{aligned}
& 2 a_{2}(\psi)=\int_{0}^{2 \pi} e^{-i \psi_{i}} d \psi(\varphi) . \\
& 6 a_{3}(\psi)=\left(\int_{0}^{2 \cdot \tau} e^{-i \varphi} d \psi(\varphi)\right)^{2}+\int_{0}^{2 \cdot \tau} e^{-i 2 \tau} d \psi(\varphi) .
\end{aligned}
$$


For every $\psi \in \Phi_{k}$, there is accordingly a unique $f \in S_{k}$, and this mapping $\Phi_{k} \rightarrow S_{k}$ is surjective. The maximum of the absolute value of coefficient $a_{p}$ in $S_{k}$ ( $p$ fixed) can thus be determined by maximizing the functional $A(\psi)=\left|a_{p}(\psi)\right|$ in $\Phi_{\boldsymbol{k}}$.

From now on, $\Phi_{k}$ is regarded as a metric space, with the metric $?$ defined by

$$
\varrho\left(\psi_{\mathbf{1}}, \psi_{\mathbf{2}}\right)=\sup _{\varphi \in I}\left|\psi_{\mathbf{1}}(\varphi)-\psi_{\mathbf{2}}(\varphi)\right| .
$$

The functional $A(\psi)$ is then continuous. This is easily seen for instance by using a connection which expresses the $a$-coefficients by means of certain $c$-coefficients (cf. TAMmI [6]):

$$
\left\{\begin{array}{l}
p(p+1) a_{p+1}=\sum_{\nu=1}^{p} c_{\nu}(p-v+1) a_{p-\nu+1}, \\
c_{p}=\int_{0}^{2_{\top}} e^{-i p \varphi} d \psi(\varphi) \quad\left(a_{1}=1 ; p=1,2, \ldots\right) .
\end{array}\right.
$$

By partial integration, we get the formula

$$
c_{p}\left(\psi_{1}\right)-c_{p}\left(\psi_{2}\right)=-i p \int_{0}^{2 \pi} e^{-i p_{\tau}}\left[\psi_{1}(\varphi)-\psi_{2}(\varphi)\right] d \phi .
$$

from which, together with the fact that the a-coefficients are polynomials of the $c$-coefficients, there follows the continuity of the functional $A$.

Let now $\Sigma_{k}$ denote the subspace of $\Phi_{k}$, which consists of step-functions. Since $\Sigma_{k}$ is dense in $\Phi_{k}$, it is tempting to see whether the functional $A$ can be maximized in $\Sigma_{k}$. In fact, if there exists

$$
\max _{\sigma \in \Sigma_{k}} A(\sigma)=A(\hat{\sigma})
$$

we have

$$
\max _{\psi \in \Phi} A(\psi)=A(\hat{\sigma})
$$

as a consequence of the continuity of $A$, and the fact that $\Sigma_{k}$ is dense in $\Phi_{k}$. In cases $p=3$ and $p=4$ we show in fact, by applying the methods of calculus, that the maximum of $A(\psi)$ in $\Sigma_{k}$ really exists, and its value can also be found. This will be done in $\$ \$ 2$ and 3 . It should be noted that this also leads to a differential equation of Schiffer-type for the extremal function.

Naturally, there can be extremal generating functions also in the complement $\Phi_{k}-\Sigma_{k}$, although our method does not give them. In the deter- 
mination of extremal $\hat{\sigma} \in \Sigma_{k}$, we use a special variation, applicable only to step-functions (cf. $2 \S$, n:o 1). Consequently our elementary necessary condition for the extremum is not applicable for characterization of possible extremal generating functions which are not step-functions.

\section{§ 2. A necessary condition for the step-function maximizing $\left|a_{3}\right|$}

\section{Variation of the points of discontinuity}

Let $\psi_{N} \in \Sigma_{k}$ be the step-function which has non-zero jumps $A_{1}, A_{2}, \ldots$, $\Delta_{N}$ at points $\varphi_{1}, \varphi_{2}, \ldots, \varphi_{N}$. By (3),

$$
\sum_{v=1}^{N} \Delta_{v}=2, \quad \sum_{v=1}^{N}\left|A_{v}\right| \leq k
$$

where $2 \leq k \leq 4$. The variation used in the following is effected by shifting the points $\varphi_{v}$ and keeping jumps $A_{v}$ fixed.

We turn to the case of $a_{3}$. It is well known that no restriction is involved in assuming $a_{3}$ to be real and positive. We introduce the notations

$$
t_{v}=e^{i \varphi_{v}} \quad(v=1,2, \ldots, N) .
$$

Then, by applying the formulae (4) to step-function $\psi_{N}$, we get

$$
\left\{\begin{array}{l}
2 a_{2}\left(\psi_{N}\right)=\sum_{v=1}^{N} \frac{I_{v}}{t_{v}}, \\
0<6 a_{3}\left(\psi_{N}\right)=\left(\sum_{v=1}^{N} \frac{\Delta_{v}}{t_{v}}\right)^{2}+\sum_{v=1}^{N} \frac{A_{v}}{t_{v}^{2}} .
\end{array}\right.
$$

By use of the abbreviation

$$
H\left(t_{1}, t_{2}, \ldots, t_{N}\right)=\left(\sum_{v=1}^{N} A_{v} t_{v}\right)^{2}+\sum_{v=1}^{N} 1_{v} t_{v}{ }^{2} .
$$

the function to be maximized is

$$
\begin{aligned}
& A_{3}\left(t_{1}, t_{2}, \ldots, t_{N}\right)=12 a_{3}\left(\psi_{N}\right) \\
& =H\left(t_{1}, t_{2}, \ldots, t_{N}\right)+H\left(t_{1}^{-1}, t_{2}^{-1} \ldots, t_{N}^{-1}\right) .
\end{aligned}
$$

For maximal $\psi_{N} \in \Sigma_{k}$ the conditions

$$
\frac{\partial A_{3}}{\partial \varphi_{\prime \prime}}=i t_{\mu} \frac{\partial A_{3}}{\partial t_{\mu}}=0 \quad\left(\prime \prime=1,2, \ldots N^{\top}\right)
$$


necessarily hold. In view of (11), (12) and the assumption $A_{\prime \prime} \neq 0$, these conditions are easily reduced to the form

$$
2 \bar{a}_{2} t_{\mu}+t_{\mu}^{2}-\frac{2 a_{2}}{t_{\mu}}-\frac{1}{t_{\mu}^{2}}=0 \quad(\mu=1, \ldots, N) .
$$

These equations are of the fourth degree with respect to $t_{u}$. The extremal step-function has thus at most four points of discontinuity $\varphi_{\mu}$, i.e.

$$
N \leq 4
$$

On the other hand

$$
2 \leq N \quad \text { for } k>2 .
$$

This is seen as follows. Let $A_{v}^{+}$denote the positive jumps and $A_{v}^{-}$the absolute values of the negative jumps of $\psi_{N}$. By (8) we have

from which

$$
\sum A_{v}^{+}-\sum A_{v}^{-}=2, \quad \sum A_{v}^{+}+\sum A_{v}^{-} \leq k .
$$

$$
\sum \Delta_{v}^{+} \leq \frac{k}{2}+1, \quad \sum A_{v}^{-} \leq \frac{k}{2}-1
$$

If $k=2, \Sigma \Delta_{v}^{-} \leq 0$. Then every $\Delta_{v}^{-}=0$, and we are dealing with the convex case, which has been completely studied by LöwnER [2]. We are here interested solely in the non-convex case, where at least one $\Delta_{v}^{-}>0$ and thus $k>2$. Both of the sets $\left\{\Delta_{v}^{+}\right\}$and $\left\{\Delta_{v}^{-}\right\}$are then non-empty, and hence $N \geq 2$.

It should finally be noted, that a function $f \in S_{k}$ generated by a stepfunction $\psi_{N} \in \Sigma_{k}$ has a polygonal image domain. The term »extremal polygon' is employed for the image-polygon of function $f \in S_{k}$, generated by an extremal step-function.

The results are collected below.

Theorem. The pre-images $t_{\mu}(\mu=1,2, \ldots, N)$ of corner points $f\left(t_{\mu}\right)$ of an extremal polygon satisfy the equation

$$
g_{3}(z)=\frac{1}{z^{2}}+\frac{2 a_{2}}{z}-2 \bar{a}_{2} z \cdots z^{2}=0
$$

or

$$
-z^{2} g_{3}(z)=z^{4}+2 \bar{a}_{2} z^{3}-2 a_{2} z-1=0 .
$$

In the non-convex cases, $2<k \leq 4$ number $N$ of the corners of the extremal polygon satisfies the inequalities

$$
2 \leq N \leq 4
$$




\section{Determination of the extremal step-function}

The necessary condition $g_{3}(z)=0$ considerably reduces the number of alternatives for the extremum case. Let us examine the different possibilities $N=4,3$ or 2 . The last case of these is the easiest, and will be treated later on. If $N=4$, all the pre-images $t_{\mu}$ are roots of the equation $g_{3}(z)=0$. If $N=3$, this equation has also a root which does not belong to the system of pre-images. Such a root of $g_{3}(z)=0$ is termed a free root of this equation. It should be noted, that the symmetric structure of (14) implies, that with $z$, also $\frac{1}{\bar{z}}$ is a root. This means that if the absolute value of the free root differs from one, then two free roots necessarily exist. Thus in the case $N=3$, all the roots of $g_{3}(z)=0$ necessarily have the absolute value one. - Apart from case $N=2$, accordingly, we have only the case in which all the roots of $g_{3}(z)=0$ have the absolute value one.

For study of the last mentioned case, the numbers $1+\Delta_{v}$ are estimated. For $k \leq 4$ there holds $\frac{k}{2}-1 \leq 1$, and thus, from (13)

$$
\begin{gathered}
A_{v}^{-} \leq \frac{k}{2}-1 \leq 1, \\
1-\Delta_{v}^{-} \geq 0 .
\end{gathered}
$$

Since obviously $1+\Delta_{v}^{+}>0$ we have generally

$$
\delta_{v}=1+\Delta_{v} \geq 0 \quad(v=1,2, \ldots N) .
$$

Expression (10) for the coefficient $a_{3}$ can now simply be rewritten in the quantities (17).

Denote the roots of $g_{3}(z)=0 \quad b y z_{1}, z_{2}, z_{3}, z_{4}$. As was stated above, in cases $N=3$ and $N=4$, all these numbers have the absolute value one: $\left|z_{\nu}\right|=1 \quad(v=1, \ldots, N)$. The pre-images $t_{\mu}$ are among these numbers $z_{v}$. If the coefficients of $(15)$ are written as symmetric polynomials of the roots $z_{v}$, we have

$$
\left\{\begin{array}{l}
2 \bar{a}_{2}=-\left(z_{1}+z_{2}+z_{3}+z_{4}\right) . \\
0=z_{1} z_{2}+z_{2} z_{3}+z_{3} z_{4}+z_{4} z_{1}+z_{2} z_{4}+z_{3} z_{1} \\
2 a_{2}=z_{1} z_{2} z_{3}+z_{2} z_{3} z_{4}+z_{3} z_{4} z_{1}-z_{4} z_{1} z_{2} . \\
-1=z_{1} z_{2} z_{3} z_{4} .
\end{array}\right.
$$

On the other hand, for real $a_{3}$ and for $a_{2}$, we get from (10) 


$$
\left\{\begin{array}{l}
6 a_{3}=\left(\sum_{1}^{N} \Delta_{v} t_{v}\right)^{2}+\sum_{1}^{N} \Delta_{v} t_{v}^{2}, \\
2 \bar{a}_{2}=\sum_{1}^{N} \Delta_{v} t_{v} .
\end{array}\right.
$$

If $N=3$, the sum $\sum_{1}^{N}$ does not include the free root of equation $g_{3}(z)=0$. It is of use to complete this sum, making the following agreement:

$$
\text { In } \sum_{1}^{N} \text { take } \Delta_{v}=0 \text { if } z_{v} \text { is a free root. }
$$

For $a_{3}$ and $a_{2}$, this allows of the following presentation, which is valid in both cases $N=3$ and $N=4$

$$
\left\{\begin{array}{l}
6 a_{3}=\left(\sum_{1}^{4} \Lambda_{v} z_{v}\right)^{2}+\sum_{1}^{4} \Delta_{v} z_{v}^{2}, \\
2 \bar{a}_{2}=\sum_{1}^{4} \Delta_{v} z_{v} .
\end{array}\right.
$$

Expression (20) of $a_{3}$ is now simplified by applying (18):

$$
\begin{aligned}
6 a_{3} & =\left(2 \bar{a}_{2}\right)^{2}+\sum_{1}^{4} \Lambda_{v} z_{v}^{2} \\
& \left.=\left(\sum_{1}^{4} z_{v}\right)^{2}+\sum_{1}^{4}\right\lrcorner_{v} z_{v}^{2} \\
& =\sum_{1}^{4}\left(1+\Lambda_{v}\right) z_{v}^{2} \\
6 a_{3} & =\sum_{1}^{4} \delta_{v} z_{v}^{2} .
\end{aligned}
$$

In view of agreement (19), condition (17) is clearly true also if $z_{v}$ is a free root; in this case $\delta_{v}=1$. Therefore, it can be deduced from $(21)$ that

$$
\begin{aligned}
0<6 a_{3} & \leq \sum_{1}^{4} \delta_{v}\left|z_{v}\right|^{2} \\
& =\sum_{1}^{4} \delta_{v}=\sum_{1}^{4}\left(1+\Delta_{v}\right) \\
& =4+\sum_{1}^{4} \Delta_{v}=6 ; \\
a_{3} & \leq 1 .
\end{aligned}
$$

From this we conclude, in view of (22), that values $N=3$ and $N=4$ do not give maximum for $a_{3}$. 
In the remaining case $N=2$ we have by virtue of (13)

$$
\Delta_{1}=\Delta_{1}^{+} \leq \frac{k}{2}+1, \Delta_{2}=-\Delta_{1}^{-} \geq 1-\frac{k}{2} \text {. }
$$

We omit the easy calculations needed to give

$$
\max a_{3}=\frac{1}{6}\left(k^{2}+2\right) .
$$

The extremal step-function, for which $a_{3}>0$, has the jumps at the points $\varphi_{1}=0, \varphi_{2}=\pi$.

\section{Differential equation for an extremal $f(z)$}

The above procedure, starting from the inequalities (17), was first applied by SCHIFFER and TAMMI [4] for maximizing $a_{3}$. It has been repeated here as a preparation for the following considerations, in which a differential equation for the extremal $f(z)$ will be derived. As stated above, the stepfunction generating $f(z)$ has $N=2, l_{1}+l_{2}=2, t_{1}=1, t_{2}=-1$.

We start from the Poisson-Stieltjes presentation (2). In the step-function case this assumes the form

$$
1+z \frac{f^{\prime \prime}(z)}{f^{\prime}(z)}=\frac{1}{2} \sum_{v=1}^{N} \frac{t_{v}+z}{t_{v}+z} \Lambda_{v} .
$$

We desire to rewrite the right side of (23) by applying the roots $z_{v}$ of the equation $g_{3}(z)=0$. It should be noted that although we are aware that the maximum case for $a_{3}$ is $N=2$, simultaneous consideration is given below to all the cases $N=2,3,4$. as an exercise for the study of $a_{4}$ in $\$ 3$. By application of the same technique to the case of $a_{4}$ there is obtained the result $(50)$, from which the ralue of $N$. which belongs to the extremal polygon, can be deduced.

According to agreement (19). formula (23) can be written in all the cases $N=2,3,4$ in the form

$$
1+z \frac{f^{\prime \prime}(z)}{f^{\prime}(z)}=\frac{1}{2} \sum_{r=1}^{4} \frac{z_{r}-z}{z_{r}-z} 1_{r} .
$$

We write the sum on the right of $(24)$ as follows

$$
\begin{gathered}
\sum_{v=1}^{4} \frac{z_{v}-z}{z_{v}-z} d_{v} \\
=\frac{1}{-z^{2} g_{3}(z)}\left[\left(z+z_{1}\right)\left(z-z_{2}\right)\left(z-z_{3}\right)\left(z-z_{4}\right) d_{1}+\ldots+\right. \\
\left.\left(z-z_{1}\right)\left(z-z_{2}\right)\left(z-z_{3}\right)\left(z+z_{4}\right) \Delta_{4}\right] .
\end{gathered}
$$


Develop the first term in the brackets by using the relations (18):

$$
\begin{aligned}
& \left(z+z_{1}\right)\left(z-z_{2}\right)\left(z-z_{3}\right)\left(z-z_{4}\right)=z^{4}-\left(-z_{1}+z_{2}+z_{3}+z_{4}\right) z^{3} \\
& \quad+\left(-z_{1} z_{2}+z_{2} z_{3}+z_{3} z_{4}-z_{4} z_{1}+z_{2} z_{4}-z_{3} z_{1}\right) z^{2} \\
& \quad-\left(-z_{1} z_{2} z_{3}+z_{2} z_{3} z_{4}-z_{3} z_{4} z_{1}-z_{4} z_{1} z_{2}\right) z-z_{1} z_{2} z_{3} z_{4} \\
& \quad=z^{4}-\left(-2 z_{1}+z_{1}+z_{2}+z_{3}+z_{4}\right) z^{3}-2 z_{1}\left(-z_{1}+z_{1}+z_{2}+z_{3}+z_{4}\right) z^{2} \\
& \quad-z_{1} z_{2} z_{3} z_{4}\left(\frac{2}{z_{1}}-\frac{1}{z_{4}}-\frac{1}{z_{1}}-\frac{1}{z_{2}}-\frac{1}{z_{3}}\right) z-z_{1} z_{2} z_{3} z_{4} \\
& =z^{4}-\left(-2 z_{1}-2 \bar{a}_{2}\right) z^{3}-2 z_{1}\left(-z_{1}-2 \bar{a}_{2}\right) z^{2}+\left(\frac{2}{z_{1}}+2 a_{2}\right) z+1 .
\end{aligned}
$$

The other three terms within brackets in (25) are treated in a similar fashion, multiplied by corresponding $\Delta_{v}$ and added. The [ ]-expression in (25) then assumes a form which can be further simplified, in view of (20), as follows:

$$
\begin{aligned}
{[]=} & \sum_{1}^{4} \Delta_{v} \cdot z^{4}+2\left(\bar{a}_{2} \sum_{1}^{4} \Delta_{v}+\sum_{1}^{4} \Delta_{v} z_{v}\right) z^{3} \\
& +2\left(2 \bar{a}_{2} \sum_{1}^{4} \Delta_{v} z_{v}+\sum_{1}^{4} \Delta_{v} z_{v}^{2}\right) z^{2}+2\left(a_{2} \sum_{1}^{4} \Delta_{v}+\sum_{1}^{4} \frac{\Delta_{v}}{z_{v}}\right) z+\sum_{1}^{4} J_{v} \\
= & 2 z^{4}+2\left(2 \bar{a}_{2}+2 \bar{a}_{2}\right) z^{3} \\
& +2\left(4 \bar{a}_{2}^{2}+6 a_{3}-4 \bar{a}_{2}^{2}\right) z^{2}+2\left(2 a_{2}+2 a_{2}\right) z+2 \\
= & 2\left(z^{4}+4 \bar{a}_{2} z^{3}+6 a_{3} z^{2}+4 a_{2} z+1\right) .
\end{aligned}
$$

The final form of equation (23) is thus

$$
1+z \frac{f^{\prime \prime}(z)}{f^{\prime}(z)}=\frac{z^{2}\left(\frac{1}{z^{2}}+\frac{4 a_{2}}{z}+6 a_{3}+4 \bar{a}_{2} z+z^{2}\right)}{z^{2} g_{3}(z)} .
$$

Summary. Let $f(z) \in S_{k}$ be the function generated by a the step-function $\psi_{N}(\varphi)$, which is extremal with respect to the coefficient $a_{3}>0$. Let the points of discontinuity of $\psi_{N}(\varphi)$ be $\varphi_{\mu}(\mu=1, \ldots, N \leq 4)$. The pre-images

$$
z=t_{\mu}=e^{i \varphi_{\mu}}
$$

of corner points $f\left(t_{\mu}\right)$ of the extremal polygon satisfy the necessary condition

$$
g_{3}(z)=\frac{1}{z^{2}}+\frac{2 a_{2}}{z}-2 \bar{a}_{2} z-z^{2}=0 .
$$


The Poisson-Stieltjes presentation for $f(z)$, generated by $\psi_{N}(\varphi)$, reads

$$
1+z \frac{f^{\prime \prime}(z)}{f^{\prime}(z)}=\frac{1}{2} \sum_{v=1}^{4} \frac{z_{v}+z}{z_{v}-z} \Lambda_{v} .
$$

Here the numbers $z_{v}$ are the roots of equation (26). If $N<4$ some of the numbers $z_{v}$ are free roots of equation $g_{3}(z)=0$. This means that they are not among the pre-images $t_{\mu}(\mu=1, \ldots, N)$ mentioned above. The numbers $\Delta_{v}$ belonging to the free roots $z_{v}$, are taken to be $=0$.

Equation (27) can also be written in the form

$$
1+z \frac{f^{\prime \prime}(z)}{f^{\prime}(z)}=\frac{G_{3}(z)}{g_{3}(z)},
$$

where

$$
G_{3}(z)=\frac{1}{z^{2}}+\frac{4 a_{2}}{z}+6 \alpha_{3}+4 \bar{a}_{2} z+z^{2} .
$$

It is emphasized that we are here led to differential equation (28), which also results from application of the method of interior variation (ScHIFFERTAmmi [4]) in class $S_{k}$. The fact that the step-function-generated extremal $f \in S_{k}$ satisfies the differential equation (28) is thus already implied by the most trivial necessary extremum conditions of calculus.

\section{§ 3 . The coefficient $a_{4}$}

\section{Differential equation for an extremal $f(z)$}

It will now be shown that the above procedure is also applicable to the next coefficient, $a_{4}$.

From (6) it is first deduced that

$$
\left\{\begin{array}{l}
24 a_{4}=c_{1}-3 c_{1} c_{2}+2 c_{3} . \\
c_{n}=\int_{0}^{2 \cdot} e^{-i n_{t}} d \psi(\psi)=\sum_{i=1}^{v} \frac{I_{v}}{t_{v}^{n}} .
\end{array}\right.
$$

We will also need the expressions of coefficients $a_{2}$ and $a_{3}$, and have

$$
\left\{\begin{aligned}
0<24 a_{4} & =\left(\sum_{1}^{N} \frac{1_{v}}{t_{v}}\right)^{3}+3 \sum_{1}^{N} \frac{1_{v}}{t_{v}} \cdot \sum_{1}^{N} \frac{1_{v}}{t_{v}^{2}}+2 \sum_{1}^{N} \frac{J_{v}}{t_{v}^{3}} \\
6 a_{3} & =\left(\sum_{1}^{N} \frac{\Delta_{v}}{t_{v}}\right)^{2}+\sum_{1}^{N} \frac{d_{v}}{t_{v}^{2}} \\
2 a_{2} & =\sum_{1}^{N} \frac{\Delta_{v}}{t_{v}}
\end{aligned}\right.
$$


For further treatment, it is of value to express the different sum-expressions in terms of the coefficients. By virtue of (31),

$$
\left\{\begin{array}{l}
\sum_{1}^{N} \frac{\Delta_{v}}{t_{v}}=2 a_{2}, \\
\sum_{1}^{N} \frac{\Delta_{v}}{t_{v}^{2}}=6 a_{3}-4 a_{2}^{2}, \\
\sum_{1}^{N} \frac{d_{v}}{t_{v}^{3}}=12 a_{4}-18 a_{2} a_{3}+8 a_{2}^{3} .
\end{array}\right.
$$

The following notations similar to the former ones will be used:

$$
\left\{\begin{array}{l}
H\left(t_{1}, t_{2}, \ldots, t_{N}\right)=\left(\sum_{1}^{N} \Delta_{v} t_{v}\right)^{3}+3 \sum_{1}^{N} \Delta_{v} t_{v} \cdot \sum_{1}^{N} \Delta_{v} t_{v}^{2}+2 \sum_{1}^{N} \Delta_{v} t_{v}^{2}, \\
A_{4}=2 \operatorname{Re}\left\{24 a_{4}\right\}=H\left(t_{1}, t_{2}, \ldots, t_{N}\right)+H\left({ }_{1}^{-1}, t_{2}^{-1}, \ldots, t_{N}^{-1}\right) .
\end{array}\right.
$$

A necessary condition for an extremal step-function can again be derived by use of the necessary extremal conditions of calculus. Hence. we necessarily have

$$
\frac{\partial A_{4}}{\partial t_{\mu}}=\frac{6 \Delta_{\mu}}{t_{\mu}}\left(3 \bar{a}_{3} t_{\mu}+2 \bar{a}_{2} t_{\mu}^{2}+t_{\mu}^{3}-\frac{3 a_{3}}{t_{\mu}}-\frac{2 a_{2}}{t_{\mu \prime}^{2}} \cdots \frac{1}{t_{\mu}^{3}}\right)=\ldots 0 .
$$

It has accordingly been found that pre-images $t_{\mu}$ of corner points $f\left(t_{\mu}\right)$ of the extremal polygon are among the roots $z_{v}(v=1, \ldots, 6)$ of the equation

$$
g_{4}(z)=\frac{1}{z^{3}}+\frac{2 a_{2}}{z^{2}}+\frac{3 a_{3}}{z}-3 \bar{a}_{3} z-2 \bar{a}_{2} z^{2}-z^{3}=0 .
$$

or

$$
-z^{3} g_{4}(z)=z^{6}+2 \bar{a}_{2} z^{5}+3 \bar{a}_{3} z^{4}-3 a_{3} z^{2}-2 a_{2} z-1=0 .
$$

Consequently

$$
2 \leq N \leq 6 \quad(2<k \leq 4)
$$

If $N<6$, then (34) has also free roots $z_{v}$ which do not belong to the preimages, denoted by $t_{\mu}$. If for a free root $z_{v}$ there holds $z_{v}=1$, then the symmetric structure of (34) indicates that together with $z_{v}$ the number 1 $\frac{1}{\bar{z}_{v}}$ is also a free root.

Again, it is desired to make use of the symmetric expressions of all the 
roots $z_{v}(v=1, \ldots, 6)$. Thus, an agreement is made which allows of replacing $\sum_{1}^{N}$ in (44) by $\sum_{1}^{6}$ :

$$
\text { In } \sum_{1}^{6} \text { take } d_{v}=0 \text { if } z_{v} \text { is a free root. }
$$

To arrive at the connections between the coefficients (31) and the symmetric expressions of $z_{v}$ mentioned, compare the left and right sides of the identity

$$
\begin{aligned}
-z^{3} g_{4}(z) & =z^{5}+2 \bar{a}_{2} z^{5}+3 \bar{a}_{3} z^{4}-3 a_{3} z^{2}-2 a_{2} z-1 \\
& =\left(z-z_{1}\right)\left(z-z_{2}\right)\left(z-z_{3}\right)\left(z-z_{4}\right)\left(z-z_{5}\right)\left(z-z_{6}\right) \\
& =z^{6}-C_{5} z^{5}+C_{4} z^{4}-C_{3} z^{3}+C_{2} z^{2}-C_{1} z+C_{0} \\
& =h_{6}(z) .
\end{aligned}
$$

Here, coefficients $C_{v}$ have the following symmetric expressions:

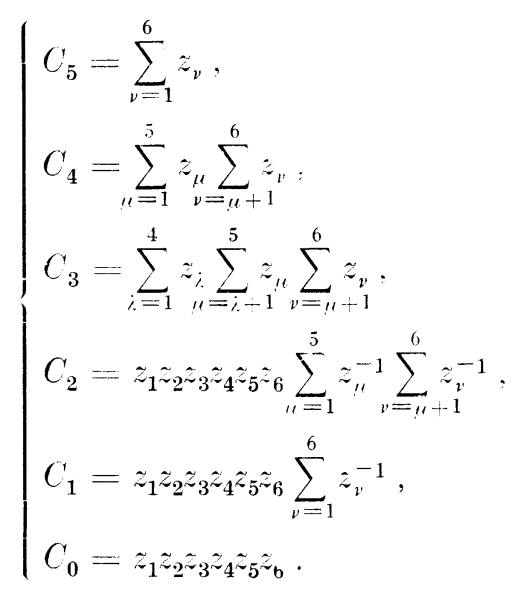

Comparison of the coefficients in (38) now indicates the comnections, which can be regarded as necessary extremum conditions

$$
\left\{\begin{aligned}
2 \bar{a}_{2} & =-C_{5}, \\
3 \bar{a}_{3} & =C_{4}, \\
0 & =C_{3}, \\
-3 a_{3} & =C_{2}, \\
-2 a_{2} & =-C_{1}, \\
-1 & =C_{0} .
\end{aligned}\right.
$$


It is readily found, that the first and fifth condition (40) are equivalent, and similarly that the second and fourth condition (40) are equivalent to each other. - The first equation (40) gives

$$
2 a_{2}=-\bar{C}_{5}=-\sum_{\nu=1}^{6} \bar{z}_{\nu} .
$$

Now, if $\left|z_{v}\right|=1$, then $\bar{z}_{v}=\frac{1}{z_{v}}$. If $\left|z_{\nu}\right| \neq 1$ there also exists a free root $z_{\mu}=\frac{1}{\bar{z}_{v}} ; \bar{z}_{\nu}=\frac{1}{z_{\mu}}$. Thus we can write (41) in the form

$$
2 a_{2}=-\sum_{v=1}^{6} \frac{1}{z_{v}} .
$$

According to the last equation (40) this is the same as

$$
2 a_{2}=z_{1} z_{2} z_{3} z_{4} z_{5} z_{6} \sum_{\nu=1}^{6} \frac{1}{z_{v}}=C_{1}
$$

and we have arrived at the fifth condition (40). - The equivalence of the second and fourth condition (40) is proved similarly. Hence, four independent necessary conditions are left:

$$
\left\{\begin{aligned}
2 \bar{a}_{2} & =-C_{5}, \\
3 \bar{a}_{3} & =C_{4}, \\
0 & =C_{3}, \\
-1 & =C_{0},
\end{aligned}\right.
$$

Our final aim is that of utilizing (40) in rewriting the Poisson-Stieltjes presentation for the extremal $f(z)$ given by the extremal $\psi_{N}(\varphi)$. In the case of $a_{4}$, the general form (23) of the presentation concerned, can be written

$$
\left.1+z \frac{f^{\prime \prime}(z)}{f^{\prime}(z)}=\frac{1}{2} \sum_{v=1}^{6} \frac{z_{v}+z}{z_{v}-z}\right\lrcorner_{v}
$$

Here again, use is made of agreement (37). - Initially, it should be noted that

$$
\begin{gathered}
\sum_{v=1}^{6} \frac{z_{v}+z}{z_{v}-z} \Delta_{v}=\frac{-1}{-z^{3} g_{4}(z)}\left[\left(z+z_{1}\right)\left(z-z_{2}\right)\left(z-z_{3}\right)\left(z-z_{4}\right)\left(z-z_{5}\right)\left(z-z_{6}\right) d_{1}\right. \\
\left.+\ldots+\left(z-z_{1}\right)\left(z-z_{2}\right)\left(z-z_{3}\right)\left(z-z_{4}\right)\left(z-z_{5}\right)\left(z+z_{6}\right) L_{6}\right] .
\end{gathered}
$$


For evaluation of the [ ]-expression, we will first rewrite its first term. Let us denote

$$
\tilde{h}_{6}(z)=\left(z+z_{1}\right)\left(z-z_{2}\right)\left(z-z_{3}\right)\left(z-z_{4}\right)\left(z-z_{5}\right)\left(z-z_{6}\right) .
$$

This polynomial is closely connected to the polynomial $h_{6}(z)$ defined by (12). We write

$$
\begin{cases}h_{6}(z)=\sum_{v=6}^{0}(-1)^{v} C_{v} z^{v} & \left(C_{6}=1\right), \\ \tilde{h}_{6}(z)=\sum_{v=6}^{0}(-1)^{v} \tilde{C}_{v} z^{v} & \left(\tilde{C}_{6}=1\right),\end{cases}
$$

and will express coefficients $\tilde{C}_{v}$ by the aid of coefficients $C_{v}$. The only alteration needed to get $\tilde{C}_{v}$ from $C_{v}$ is to change the sign of $z_{1}$ in $C_{v}$. In simplification of the expression of $C_{2}$ we need the connection

$$
\bar{C}_{5}=\sum_{v=1}^{6} \frac{1}{z_{v}},
$$

which follows from the considerations connected with (41). Consequently:

$$
\begin{aligned}
& \tilde{C}_{5}=-2 z_{1}+C_{5}, \\
& \tilde{C}_{4}=-2 z_{1} \sum_{\nu=2}^{6} z_{\nu}+C_{4}=-2 z_{1}\left(-z_{1}+C_{5}\right)+C_{4}, \\
& \tilde{C}_{3}=-2 z_{1} \sum_{\mu=2}^{5} z_{\mu} \sum_{\nu=\mu+1}^{6} z_{v}+C_{3} \\
& =-2 z_{1}\left[-z_{1} \sum_{\nu=2}^{6} z_{v}+\sum_{\mu=1}^{5} z_{\mu} \sum_{\nu=\mu+1}^{6} z_{\nu}\right]+C_{3} \\
& =-2 z_{1}\left[-z_{1}\left(-z_{1}+C_{5}\right)+C_{4}\right]+C_{3} \text {. } \\
& \tilde{C}_{2}=-z_{1} z_{2} z_{3} z_{4} z_{5} z_{6}\left(-2 z_{1}^{-1} \sum_{v=2}^{6} z_{v^{-1}}^{-1}+\sum_{\mu=1}^{5} z_{\mu}^{-1} \sum_{v=\mu+1}^{6} z_{v}^{-1}\right) \\
& =-z_{1} z_{2} z_{3} z_{4} z_{5} z_{6}\left[-2 z_{1}^{-1}\left(-z_{1}^{-1}+\sum_{v=1}^{6} z_{v}^{-1}\right)+\sum_{n=1}^{5} z_{n}^{-1} \sum_{v=11}^{6} z_{v}^{-1}\right] \\
& =-C_{0}\left[-2 z_{1}^{-1}\left(-z_{1}^{-1}+\bar{C}_{5}\right)\right]-C_{2} \text {, } \\
& \tilde{C}_{1}=-z_{1} z_{2} z_{3} z_{4} z_{5} z_{6}\left(-2 z_{1}^{-1}+\sum_{r=1}^{6} z_{v}^{-1}\right) \\
& =C_{0} \cdot 2 \approx_{1}^{-1}-C_{1}, \\
& \tilde{C}_{0}=\ldots C_{0} \text {. }
\end{aligned}
$$


Hence quite a simple expression for $\tilde{h}_{6}(z)$ results, when still applying the necessary conditions (40):

$$
\begin{aligned}
\tilde{h}_{6}(z)= & z^{6}-\left(-2 z_{1}+C_{5}\right) z^{5}+\left[-2 z_{1}\left(-z_{1}+C_{5}\right)+C_{4}\right] z^{4} \\
& +\left\{2 z_{1}\left[-z_{1}\left(-z_{1}+C_{5}\right)+C_{4}\right]+C_{3}\right\} z^{3} \\
& +\left\{-C_{0}\left[-2 z_{1}^{-1}\left(-z_{1}^{-1}+\bar{C}_{5}\right)\right]-C_{2}\right\} z^{2} \\
& -\left(C_{0} \cdot 2 z_{1}^{-1}-C_{1}\right) z-C_{0} \\
= & z^{6}+\left(2 z_{1}+2 \bar{a}_{2}\right) z^{5} \\
& +\left(2 z_{1}^{2}+4 \bar{a}_{2} z_{1}+3 \bar{a}_{3}\right) z^{4}+\left(2 z_{1}^{3}+4 \bar{a}_{2} z_{1}^{2}+6 \bar{a}_{3} z_{1}\right) z^{3} \\
& +\left(2 z_{1}^{-2}+4 a_{2} z_{1}^{-1}+3 a_{3}\right) z^{2}+\left(2 z_{1}^{-1}+2 a_{2}\right) z+1 .
\end{aligned}
$$

The first term in the [ ]-expression of (44) is thus

$$
\begin{aligned}
& h_{6}(z) \Delta_{1}=\Delta_{1} z^{6}+2\left(z_{1} \Delta_{1}+\bar{a}_{2} \Delta_{1}\right) z_{5} \\
& +\left(2 z_{1}^{2} \Delta_{1}+4 \bar{a}_{2} z_{1} \Delta_{1}+3 \bar{a}_{3} \Delta_{1}\right) z^{4}+2\left(z_{1}^{3} \Delta_{1}+2 \bar{a}_{2} z_{1}^{2} \Delta_{1}+3 \bar{a}_{3} z_{1} \Delta_{1}\right) z^{3} \\
& +\left(2 \frac{\Delta_{1}}{z_{1}^{2}}+4 a_{2} \frac{\Delta_{1}}{z_{1}}+3 a_{3} \Delta_{1}\right) z^{2}+2\left(\frac{\Delta_{1}}{z_{1}}+a_{2} \Delta_{1}\right) z+\Delta_{1} .
\end{aligned}
$$

By cyclic permutation, and addition the [ ]-expression of (44) assumes the form

$$
\begin{aligned}
{[] } & =\sum_{1}^{6} A_{v} \cdot z^{6}+2\left(\sum_{1}^{6} z_{v} A_{v}+\bar{a}_{2} \sum_{1}^{6} A_{v}\right) z^{5} \\
& +\left(2 \sum_{1}^{6} z_{v}^{2} A_{v}+4 \bar{a}_{2} \sum_{1}^{6} z_{v} A_{v}+3 \bar{a}_{3} \sum_{1}^{6} A_{v}\right) z^{4} \\
& +2\left(\sum_{1}^{6} z_{v}^{3} A_{v}+2 \bar{a}_{2} \sum_{1}^{6} z_{v}^{2} A_{v}+3 \bar{a}_{3} \sum_{1}^{6} z_{v} \Delta_{v}\right) z^{3} \\
& +\left(2 \sum_{1}^{6} \frac{\Delta_{v}}{z_{v}^{2}}+4 a_{2} \sum_{1}^{6} \frac{\Delta_{v}}{z_{v}}+3 a_{3} \sum_{1}^{6} A_{v}\right) z^{2} \\
& +2\left(\sum_{1}^{6} \frac{\Delta_{v}}{z_{v}}+a_{2} \sum_{1}^{6} A_{v}\right) z+\sum_{1}^{6} A_{v} .
\end{aligned}
$$

All the sums $\sum_{1}^{6}$ can now be expressed in the coefficients $a_{v}$ by using expressions (32) and agreement (37). This finally gives 


$$
\begin{aligned}
{[]=} & 2 z^{2}+2\left(2 \bar{a}_{2}+\bar{a}_{2} \cdot 2\right) z^{5} \\
& +\left[2\left(6 \bar{a}_{3}-4 \bar{a}_{2}^{2}\right)+4 \bar{a}_{2} \cdot 2 \bar{a}_{2}+3 \bar{a}_{3} \cdot 2\right] z^{4} \\
& +2\left[12 a_{4}-18 \bar{a}_{2} \bar{a}_{3}+8 \bar{a}_{2}^{3}+2 \bar{a}_{2}\left(6 \bar{a}_{3}-4 \bar{a}_{2}^{2}\right)+3 \bar{a}_{3} \cdot 2 \bar{a}_{2}\right] z^{3} \\
& +\left[2\left(6 a_{3}-4 a_{2}^{2}\right)+4 a_{2} \cdot 2 a_{2}+3 a_{3} \cdot 2\right] z^{2} \\
& +2\left(2 a_{2}+a_{2} \cdot 2\right) z+2 \\
= & 2 z^{6}+8 \bar{a}_{2} z^{5}+18 \bar{a}_{3} z^{3}+24 a_{4} z^{3}+18 a_{3} z^{2}+8 a_{2} z+2 .
\end{aligned}
$$

The right side of (43) has thus assumed the form

$$
\begin{aligned}
\frac{1}{2} \sum_{v=1}^{6} \frac{z_{v}+z}{z_{v}-z} A_{v}= & \left.\frac{1}{2} \frac{1}{z^{3} g_{4}(z)} \mid\right] \\
& z^{3}+4 \bar{a}_{2} z^{2}+9 \bar{a}_{3} z+12 a_{4}+\frac{9 a_{3}}{z}+\frac{4 a_{2}}{z^{2}}+\frac{1}{z^{3}} \\
& =\frac{g_{4}(z)}{}
\end{aligned}
$$

Theorem. Let $f(z) \in S_{k}$ be an extremal function for the coefficient $a_{4}>0$, which is generated by a step-function $\psi_{N}(\varphi)$ with the points of discontinuity $\varphi_{\mu}(\mu=1, \ldots, N)$. The pre-images $t_{\mu}=e^{i_{\mu} \mu}$ of the corner points of the extremal polygon satisfy the necessary condition

$$
g_{4}(z)=\frac{1}{z^{3}}+\frac{2 a_{2}}{z^{2}}+\frac{3 a_{3}}{z}-3 \bar{a}_{3} z-2 \bar{a}_{2} z^{2}-z^{3}=0 .
$$

The Poisson-Stieltjes presentation for $f(z)$ is

$$
1+z \frac{f^{\prime \prime}(z)}{f^{\prime}(z)}=\frac{1}{2} \sum_{v=1}^{6} \frac{z_{v}+z}{z_{v}-z} d_{v} .
$$

Here $z_{v}$ are the roots of equation (48) and for the corresponding $\mathbf{L}_{v}$, agreement (37) holds. Equation (49) can be written in the form

$$
1+z \frac{f^{\prime \prime}(z)}{f^{\prime}(z)}=\frac{\sigma_{4}(z)}{g_{4}(z)},
$$

where

$$
a_{4}(z)=\frac{1}{z^{3}}+\frac{4 a_{2}}{z^{2}}+\frac{9 a_{3}}{z}+12 a_{4}+9 \bar{a}_{3} z+4 \bar{a}_{2} z^{2}+z^{3}
$$

\section{On determination of $\max \mid a_{4}$}

Result (50), which was proved to hold for every extremal function $f(z)$ generated by step-functions is the same as that obtained from formulae well known in the theory of variation of univalent functions [4]. We con- 
sider it important to have arrived at the result (50) without any application of the variational theory mentioned.

In [5] Shiffer and TAmmi, were able to determine a sharp upper bound for $\left|a_{4}\right|$. This was done by starting from the Poisson-Stieltjes presentation (49), and the necessary condition (50). Comparison of the right sides of these equations provides the necessary conditions (15) and (16) of [5]. From these, maximalization follows laboriously by a proper use of Schwarz's inequality. - It should be remarked, that on the ground of the present paper, the conditions (15) and (16) of [5] appear to be direct consequences of the necessary conditions (42). Thus our conclusion is:

The sharp upper bound of $\left|a_{\mathbf{4}}\right|$ in the class $S_{k}$ follows already from the necessary condition (48).

\section{References}

[1] Leнto, O.: On the distortion of conformal mappings with bounded boundary rotation. - Ann. Acad. Sci. Fenn. Ser. A I, n:o 124 (1952).

[2] LöwnER, K.: Untersuchungen über die Verzerrung bei konformen Abbildungen des Einheitskreises $|z|<1$, die durch Funktionen mit nicht verschwindender Ableitung geliefert werden. - Ber. der Kön. Sächsischen Gesellschaft der Wiss. zu Leipzig, 26 (1917).

[3] PaAtero, V.: Über die konforme Abbildung von Gebieten, deren Ränder von beschränkter Drehung sind. - Ann. Acad. Sci. Fenn. Ser. A. 33, n:o 9 (1931).

[4] Schiffer, M. - TAmmi, O.: A method of variations for functions with bounded boundary rotation. - Journal d'Analyse Mathématique, vol. XVII, pp. $109-144$ (1966).

[5] - - - - On the fourth coefficient of univalent functions with bounded boundary rotation. - Ann. Acad. Sci. Fenn. Ser. A I, n:o 396 (1967).

[6] TаммI, O.: On certain combinations of the coefficients of schlicht functions. Ibid., n:o 140 (1952). 